\title{
Comparison of the effects of the oral anticancer platinum(IV) complexes oxoplatin and metabolite cis-diammine-tetrachlorido-platinum(IV) on global gene expression of $\mathrm{NCl}-\mathrm{H} 526$ cells
}

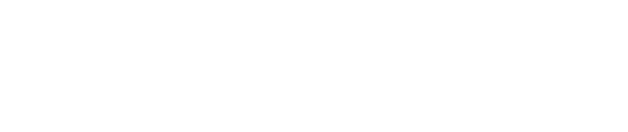

\section{Ulrike Olszewski \\ Ernst Ulsperger \\ Klaus Geissler \\ Gerhard Hamilton}

Ludwig Boltzmann Institute of Clinical Oncology and Photodynamic Therapy, Ludwig Boltzmann Cluster of Translational Oncology, Vienna, Austria
Correspondence: Gerhard Hamilton LBC of Translational Oncology, c/o Balderichgasse 26/I3, A-II 70 Vienna, Austria

Tel +43 | 404006627

$\mathrm{Fax}+43$ । 404006627

Email gerhard.hamilton@toc.lbg.ac.at

\begin{abstract}
Platinum(IV) coordination complexes like oxoplatin (cis, cis, trans-diamminedichlorido-dihydroxido-platinum[IV]) show high stability and therefore can be utilized orally for outpatient care. Although oxoplatin is capable of binding directly to DNA after prolonged incubation, platinum(IV) agents are considered to be largely inert prodrugs that are converted to highly cytotoxic platinum(II) compounds by reducing substances, enzymes, or microenvironmental conditions. Reaction of oxoplatin with $0.1 \mathrm{M}$ hydrogen chloride mimicking gastric acid yields cis-diammine-tetrachlorido-platinum(IV) (DATCP[IV]), which exhibits two-fold increased activity. The presence of chlorides as ligands in the axial position results in a high reduction potential that favors transformation to platinum(II) complexes. In this study, the intracellular effect of the highly reactive tetrachlorido derivative was investigated in comparison with an equipotent dose of cisplatin. Genome-wide expression profiling of NCI-H526 small cell lung cancer cells treated with these platinum species revealed clear differences in the expression pattern of affected genes and concerned cellular pathways between DATCP(IV) and cisplatin. Application of DATCP(IV) resulted in extensive downregulation of protein and ATP synthesis, cell cycle regulation, and glycolysis, in contrast to cisplatin, which preferentially targeted glutathione conjugation, pyruvate metabolism, citric acid cycle, and the metabolism of amino acids and a range of carbohydrates. Thus, the oxoplatin metabolite DATCP(IV) constitutes a potent cytotoxic derivative that may be produced by gastric acid or acidic areas prevailing in larger solid tumors, depending on the respective pharmaceutical formulation of oxoplatin. Furthermore, DATCP(IV) exhibits intracellular effects that are clearly different from the expected reduced product cisplatin(II). In conclusion, activation of the platinum(IV) complex oxoplatin seems to involve the generation of a cytotoxic six-coordinate species, dependent on prevailing conditions, and its effects need to be considered in addition to the effects of the potential final platinum(II) product.
\end{abstract}

Keywords: platinum, oxoplatin, metabolites, small cell lung cancer, cell line, gene expression, microarray

\section{Introduction}

Cisplatin (cis-diammine-dichlorido-platinum[II]) was established as a drug that is active against a range of malignancies, including testicular, ovarian, head and neck, bladder, esophageal, and small cell lung cancer (SCLC). ${ }^{1,2}$ However, tumors like colon and breast cancer show limited sensitivity, and cisplatin-induced resistance and severe side effects are frequently observed. ${ }^{3}$ Second-generation platinum(II)-based drugs include carboplatin, which has similar anticancer activity but fewer side effects than 
cisplatin, and oxaliplatin, which exhibits cytotoxicity against cisplatin-refractory cancer types like colorectal tumors. ${ }^{4}$ In an attempt to develop platinum drugs with enhanced stability that are suitable for oral application, axial ligands were introduced, yielding platinum(IV) coordination complexes with increased kinetic inertness and reduced reactivity, resulting in decreased degradation in the bloodstream, lower toxicity, and partial efficacy in cisplatin-resistant tumor cell lines. ${ }^{5,6}$ Thus, pharmacokinetic properties of these agents can be fine-tuned by modification of the axial substituents. Satraplatin (bisacetato-ammine-dichlorido-cyclohexylamine-platinum[IV]; JM 216), an orally applicable cisplatin analog, constitutes one of the first third-generation platinum complexes that has undergone clinical trials with limited success. ${ }^{7}$

Because it is generally accepted that reduction of the platinum(IV) central atom has to occur prior to binding to target DNA, these molecules are believed to represent prodrugs. ${ }^{8,9}$ The following reduction produces platinum(II) species that bind to DNA and lead to the formation of intraand/or interstrand adducts, which results in cell cycle arrest in the G2M phase and cell death.,10 Cellular reducing substances such as ascorbic acid and thiol-containing species like metallothioneins and glutathione are regarded as activators of platinum(IV) prodrugs. ${ }^{11,12}$

A further orally applicable platinum(IV) anticancer drug that is currently under development is oxoplatin, which was synthesized for the first time by Chugaev and Khlopin in the Russian Federation in 1927 (Figure 1). ${ }^{13}$ Its cytotoxic activity

A

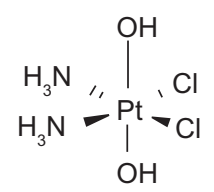

C

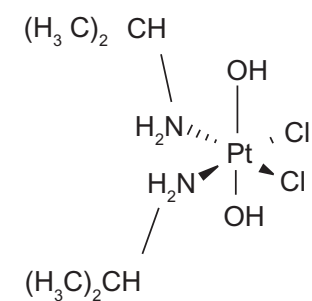

Figure I Chemical structures of the platinum compounds used or discussed (iproplatin and ormaplatin) in the present study. The full chemical formulas are cis, cis, trans-diammine-dichlorido-dihydroxido-platinum(IV) for oxoplatin, cis-diamminetetrachlorido-platinum(IV) for DATCP(IV), cis-dichloro-trans-dihydroxy-bis-isop ropylamine-platinum(IV) for iproplatin, and tetrachloro-(D,L-trans)-l,2-diaminocyclohexane-platinum(IV) for ormaplatin, respectively. was not demonstrated in rat tumor models until $1977 .{ }^{14}$ Presnov et al compared antitumor and pharmacokinetic properties of oxoplatin with those of cisplatin. Therapeutic and maximum tolerated doses were 10-fold higher for oxoplatin than for cisplatin. Additionally, oxoplatin exhibited a prolonged therapeutic effect, antimetastatic activity, and inhibition of tumor growth similar to, or even better than, cisplatin. Oxoplatin can bind directly to DNA; however, this process is so slow that it is of minimal biological relevance. ${ }^{15}$ The in vitro cytotoxicity of oxoplatin and its possible activation by reduction through reaction with hydrogen chloride $(\mathrm{HCl})$ and ascorbic acid were investigated in a previous study. ${ }^{16}$ Because oxoplatin may represent a prodrug of cisplatin, the effects of both platinum drugs on gene expression patterns of a sensitive cell line were compared using microarrays for genome-wide expression analysis. ${ }^{16}$

The antiproliferative activity of cisplatin was not affected by previous incubation with $0.1 \mathrm{M} \mathrm{HCl}$; however, these highly acidic conditions resulted in two-fold enhanced cytotoxicity for oxoplatin due to its conversion to $c i s$-diammine-tetrachloridoplatinum(IV) (DATCP[IV]) (Figure 1). Similar platinum(IV) complexes, namely iproplatin and ormaplatin (also termed tetraplatin) (Figure 1), had been investigated in clinical trials that were abandoned because of high toxicity of ormaplatin and low activity of iproplatin. ${ }^{17,18}$ Both agents are prodrugs that are converted to platinum(II) species with increased activity via reduction that takes place rapidly for ormaplatin and slowly for iproplatin. ${ }^{19}$ A breakthrough was achieved with bis(carboxylato)-platinum(IV) analogs, showing reduction potentials situated between drugs with either chloride or hydroxide axial ligands. ${ }^{20}$ Two bis(carboxylato)-platinum(IV) compounds, namely satraplatin and LA-12 (bis[acetato]adamantylamine-[ammine]-dichlorido-platinum[IV]), have been investigated in clinical trials. ${ }^{21}$ Satraplatin has two acetate moieties and needs to be hydrolyzed and subsequently reduced in order to exert an anticancer effect.

The presence of axial chloride or acetate ligands results in a slightly higher lipophilicity compared with the platinum(II) analog, whereas hydroxide substituents lead to significantly lower lipophilicity. ${ }^{22,23}$ According to these data, the tetrachlorido metabolite of oxoplatin, DATCP(IV), is expected to be reduced immediately in the cytoplasm and its actual cytotoxic effects to be caused by the main resulting reduction product cisplatin. In order to test this assumption, the effects of cisplatin and DATCP(IV) on global gene expression of the platinum-sensitive SCLC cell line NCI-H526 were investigated by microarrays in the present study. 


\section{Materials and methods Chemicals and cell line}

Unless otherwise noted, all chemicals and solutions were obtained from Sigma-Aldrich (St Louis, MO). Oxoplatin and DATCP(IV) were synthesized according to standard procedures by Chiracon (Luckenwalde, Germany) and kindly provided by IPSS (Berlin, Germany). The NCI-H526 cell line was obtained from the American Tissue Culture Collection (ATCC, Manassas, VA). Cells were cultured in RPMI-1640 medium supplemented with 10\% fetal bovine serum (Seromed, Berlin, Germany), $4 \mathrm{mM}$ glutamine, and antibiotics.

All compounds were prepared as stock solutions of $2 \mathrm{mg} / \mathrm{mL}$ in DMSO and aliquots stored at $-20^{\circ} \mathrm{C}$.

\section{Cell proliferation assay}

Cells were harvested, counted, and distributed into the wells of flat-bottomed 96-well microtiter plates at a density of $1 \times 10^{4}$ cells/well in $100 \mu \mathrm{L}$ medium. A total of $100 \mu \mathrm{L}$ of appropriate dilutions of test compounds were added to each well, and the plates were incubated under tissue culture conditions for 4 days. Stock solutions of the compounds were diluted more than 100 -fold for use in assays. Solvent control wells were included in all tests. Dose-response curves were obtained by assessment of cell growth at twofold drug dilutions in triplicate and used for calculation of the $\mathrm{IC}_{50}$ values. Cell proliferation was quantified using a modified tetrazolium dye assay (MTT; EZ4U, Biomedica, Vienna, Austria).

\section{Genome-wide gene expression analysis}

Lysates of $30 \times 10^{6}$ cells (extraction buffer: $4 \mathrm{M}$ guanidine isothiocyanate, $0.5 \%$ sodium N-lauroyl sarcosinate, $10 \mathrm{mM}$ EDTA, $5 \mathrm{mM}$ sodium citrate, $100 \mu \mathrm{M} \beta$-mercaptoethanol; 30 minutes, $4^{\circ} \mathrm{C}$ ) were added to cesium trifluoroacetate and centrifuged $\left(46,000 \mathrm{rpm}, 15^{\circ} \mathrm{C}, 20\right.$ hours). Supernatant containing DNA was removed and RNA precipitated with ice-cold $96 \%$ ethanol. Pellets were washed and, following removal of ethanol, resuspended in sterile water. RNA content was measured photometrically.

Gene expression analysis was performed using the Applied Biosystems Human Genome Survey Microarray V2.0 (Applied Biosystems, Foster City, CA) according to the manufacturer's instructions. Therefore, $2-5 \mu \mathrm{g}$ mRNA (20-50 $\mu \mathrm{g}$ total RNA) was reversely transcribed (RT) to firststrand cDNA (MyCycler thermocycler, BioRad, Hercules, CA). The RT mixture was labeled on ice and purified according to the manufacturer's instructions for the Applied
Biosystems 1700 RT Labeling Kit. Hybridization of cDNA and microarray analysis (Applied Biosystems 1700) was carried out following the manufacturer's chemiluminescence detection kit protocol. Data for each cell line $(n=2)$ were filtered, normalized, and log2-transformed, before further processing was carried out using Microsoft Excel software/ SAM (false discovery rate of $10 \%$; Statistical Analysis of Microarray, Stanford University, Stanford, CA). ABI 1700 gene identities can be accessed via the Panther classification system (www.pantherdb.org). ID mapping, pathway assignment, and over-representation analysis of cellular pathways were performed using the Reactome version 35 database (www.reactome.org).

\section{Results}

NCI-H526 SCLC cells were treated with either $4.1 \mu \mathrm{M}$ cisplatin or $1.35 \mu \mathrm{M}$ DATCP(IV) for 3 days, which resulted in cell cycle arrest but a cell viability of over $93 \%$ (data not shown). Under these conditions, decreases in mitochondrial activity were detectable on day 4 in chemosensitivity assays in the previous study. ${ }^{16}$ Cells were harvested and counted and lysates prepared for genome-wide expression analysis. The 40 genes found to be either overexpressed or downregulated to the largest extent in treated NCI-H526 cell in response to cisplatin or DATCP(IV), respectively, in comparison with untreated medium controls, are listed in Table 1. These data demonstrate that the majority of genes are clearly differentially affected by the two compounds. The folate receptor 1 (FOLR1) is the gene that is upregulated by both platinum drugs. Similarly, analysis of the 40 most downregulated genes revealed no concordance.

Because these gene expression differences in NCIH526 cells may be quantitative rather than qualitative, all genes that were downregulated or upregulated more than four-fold, respectively, in response to one of the two platinum complexes were checked for over-representation in pathway analysis employing the Reactome database. In the case of cisplatin, over-represented pathways involving downregulated genes included glutathione conjugation, pyruvate metabolism, citric acid cycle, and cellular signal transduction, as well as metabolism of a range of carbohydrates and amino acids (Table 2). Corresponding upregulated pathways comprised metabolic regulation, energy metabolism, and distinct signaling cascades, including mediators like glucagon, phospholipase C (PLC), calmodulin ( $\mathrm{CaM}$ ), adenylate cyclase, and cAMP-responsive element binding protein (CREB). For DATCP(IV), downregulation of genes participating in protein synthesis and turnover, replication, transcription, respiration, 
Table I Alterations of gene expression in platinum drug-treated $\mathrm{NCl}-\mathrm{H} 526$ cells. The 40 genes exhibiting highest down- or upregulated expression in treated $\mathrm{NCl}-\mathrm{H} 526$ small cell lung cancer cells in response to cisplatin or DATCP(IV), respectively, compared with untreated cells (fold $\Delta$ : $\mathrm{n}$-fold change in gene expression treated/untreated cells)

\begin{tabular}{|c|c|c|c|c|c|c|c|}
\hline $\begin{array}{l}\text { Cisplatin } \\
\text { upregulated }\end{array}$ & Fold $\Delta$ & $\begin{array}{l}\text { DATCP(IV) } \\
\text { upregulated }\end{array}$ & Fold $\Delta$ & $\begin{array}{l}\text { Cisplatin } \\
\text { downregulated }\end{array}$ & Fold $\Delta$ & $\begin{array}{l}\text { DATCP(IV) } \\
\text { downregulated }\end{array}$ & Fold $\Delta$ \\
\hline$\overline{A S C L I}$ & 200.5 & MPP4 & 946.7 & GSTPI & 0.002 & TUBA & 0.007 \\
\hline MAGEA4 & 72.9 & MMP26 & 56.2 & AVIL & 0.005 & RPLP2 & 0.008 \\
\hline MAGEC2 & 38.9 & DKK2 & 46.7 & LOC5II6I & 0.007 & TMSBIO & 0.012 \\
\hline PAGE-5 & 35.3 & FBXLI3 & 42.9 & BASPI & 0.008 & STMNI & 0.013 \\
\hline ISLI & 24.7 & СКМТ2 & 38.6 & PRSS3 & 0.011 & RPL4I & 0.015 \\
\hline DCX & 22.9 & EYAI & 32.1 & TMPRSS3 & 0.012 & RPLI7 & 0.015 \\
\hline XAGEID & 17.5 & BCL6 & 32.0 & ILI3RAI & 0.015 & XRCC5 & 0.016 \\
\hline RBPI & 15.5 & NPY & 27.7 & CD9 & 0.015 & RPS2I & 0.020 \\
\hline GS3955 & 14.9 & OPB2B & 22.7 & DDXI & 0.015 & UBE4A & 0.020 \\
\hline DNER & 14.8 & DGKQ & 22.5 & RNPCI & 0.015 & TUBA4 & 0.023 \\
\hline SERPINB8 & 13.9 & FOLRI & 22.4 & SPOCKI & 0.015 & RPSI9 & 0.027 \\
\hline COLIA2 & 13.3 & HNMT & 22.1 & NKX6-I & 0.017 & CALM2 & 0.028 \\
\hline STMN2 & 13.2 & SCNNID & 20.6 & PTPNI8 & 0.018 & DGKZ & 0.030 \\
\hline FLJ32942 & 12.9 & EDIL3 & 19.8 & SLCIA5 & 0.018 & RASGEF & 0.031 \\
\hline TUBA3E & II.I & $\mathrm{EMCN}$ & 19.7 & THYI & 0.018 & UBB & 0.031 \\
\hline KLHLI & 11.0 & KIF9 & 18.9 & FABP5 & 0.020 & RPLI3A & 0.033 \\
\hline НT02I & 10.7 & CYP2CI8 & 18.0 & CSNKIGI & 0.021 & RPSSI 2 & 0.036 \\
\hline SYTI & 9.8 & WNTI6 & 17.7 & COL27AI & 0.021 & HISTIH4C & 0.036 \\
\hline SLC43A3 & 8.9 & BFSPI & 17.1 & RGSI3 & 0.024 & HNRPA2BI & 0.037 \\
\hline FOXGIB & 8.8 & SLC3AI & 14.5 & NMU & 0.026 & $\mathrm{AHCY}$ & 0.037 \\
\hline PNMA5 & 8.7 & HAVCRI & 14.3 & AZGPI & 0.029 & RPL26 & 0.039 \\
\hline PAGE3 & 7.9 & HUSIB & 14.2 & VILI & 0.029 & SLC25A5 & 0.040 \\
\hline DDC & 7.3 & PTGFR & 13.4 & ELF3 & 0.030 & ATPIAI & $0.04 I$ \\
\hline ELAVL4 & 7.1 & KCNKI & 12.9 & SCGB2AI & 0.031 & cox7C & 0.042 \\
\hline SIX3 & 6.9 & IFNAI4 & 12.4 & IDI & 0.032 & RPSI6 & 0.044 \\
\hline CaMKIINalpha & 6.7 & ADAM33 & 12.3 & TNFSF8 & 0.033 & TPTI & 0.045 \\
\hline NCALD & 6.5 & SLC4A4 & 12.1 & NMTI & 0.033 & MORF4L & 0.046 \\
\hline KCNMB2 & 6.4 & $\mathrm{CDHI} 3$ & 11.9 & MLL & 0.033 & RPL4I & 0.048 \\
\hline MS4A8B & 6.2 & PPEF2 & 11.6 & FASN & 0.034 & TMSB4X & 0.048 \\
\hline CROT & 6.2 & GDF8 & 11.3 & MAZ|KIF22 & 0.035 & RPLI4 & 0.049 \\
\hline АРОВЕС $3 B$ & 6.1 & DMPI & 10.7 & LY6E & 0.036 & HNRPAI & 0.049 \\
\hline NKX2-I & 5.9 & LATS2 & 10.5 & MYOIO & 0.037 & HDGF & 0.051 \\
\hline FOLRI & 5.7 & $\mathrm{ARCH}$ & 10.3 & MFNG & 0.038 & SNRPF & 0.052 \\
\hline XAGE3 & 5.6 & SLC35B4 & 10.2 & JAKI & 0.038 & ACTB & 0.055 \\
\hline PKIB & 5.5 & TGM5 & 10.2 & EN2 & 0.039 & UCHLI & 0.057 \\
\hline MAGECI & 5.4 & HTR3E & 9.9 & RASD2 & 0.039 & NGFRAPFI & 0.058 \\
\hline LPL & 5.4 & SPACA4 & 9.5 & SYT7 & 0.040 & $\mathrm{SMT} 3 \mathrm{H} 2$ & 0.059 \\
\hline GRP & 5.3 & PRX & 9.1 & HNRPAO & 0.040 & BTF3 & 0.060 \\
\hline GBA3 & 5.2 & DNAJB5 & 9.0 & VAMP2 & 0.041 & RPL3I & 0.060 \\
\hline NKX2-2 & 4.8 & RFPLI & 8.9 & RGL & 0.041 & PPIA & 0.060 \\
\hline
\end{tabular}

cell cycle regulation, p53-dependent and -independent damage response, glycolysis/gluconeogenesis, and others were found (Table 3). Upregulated transcripts included those involved in metabolism of xenobiotics, metal ion transport, H-RAS activation, and cell junction organization. According to the Reactome database, with the single exception of the "metabolism of carbohydrates" (REACT_474/1383/1520) partially overlapping pathways, none of the processes mostly affected by either cisplatin and DATCP(IV) was the same, which indicates important differences in their mechanisms of cytotoxicity.

\section{Discussion}

The development of orally applicable platinum-based anticancer drugs is currently being intensively pursued in order to avoid intravenous administration, allowing for outpatient care. ${ }^{1,2}$ Among the first oral platinum coordination complexes established are picoplatin(II) and satraplatin(IV), which have shown promise in preclinical and clinical trials but have so far failed to gain approval. ${ }^{4}$ Platinum(IV) compounds are considered prodrugs that are converted to their cytotoxically active platinum(II) forms primarily at the target site. ${ }^{23}$ Oxoplatin is converted to platinum(II) 
Table 2 Over-representation pathway analysis of genes more than four-fold down- or upregulated in $\mathrm{NCl}-\mathrm{H} 526$ small cell lung cancer cells treated with cisplatin. Gene expression was assessed using Applied Biosystems Human Genome Survey Microarray V2.0, and data were analyzed using the Reactome database

\begin{tabular}{|c|c|c|}
\hline$P$ value & Identifier event & Name of this event \\
\hline \multicolumn{3}{|c|}{ Cisplatin downregulated } \\
\hline 0.0006 & REACT_I84|4 & Dephosphorylation of NCAMI bound pFyn \\
\hline 0.0033 & REACT_6926 & Glutathione conjugation \\
\hline 0.0047 & REACT_22296 & Upregulation of cytosolic proteins by activated PPARA \\
\hline 0.0057 & REACT_I046 & Pyruvate metabolism and citric acid (TCA) cycle \\
\hline 0.0086 & REACT_25287 & The $\mathrm{Na}^{+} / \mathrm{K}^{+}$-transporting ATPase \\
\hline 0.0109 & REACT_6854 & Glutathione conjugation of cytosolic substrates \\
\hline 0.0109 & REACT_I4820 & Metabolism of polyamines \\
\hline 0.0135 & REACT_34 & Ethanol oxidation \\
\hline 0.0163 & REACT_I2527 & EGFR non-clathrin mediated endocytosis \\
\hline 0.0163 & REACT_I8333 & Recruitment of FAK to NCAMI:Fyn in lipid rafts \\
\hline 0.0187 & REACT_474 & Metabolism of carbohydrates \\
\hline 0.0226 & REACT_I 2387 & Sprouty sequesters $\mathrm{Cb}$ away from active EGFR \\
\hline 0.0226 & REACT_I8259 & SOS binds Grb2 bound to pFAK:NCAMI \\
\hline 0.0251 & REACT_I3 & Metabolism of amino acids and derivatives \\
\hline 0.0417 & REACT_207I & Pyruvate metabolism \\
\hline 0.0461 & REACT_I 785 & Citric acid cycle (TCA cycle) \\
\hline 0.0461 & REACT_I2495 & Assembly in clathrin-coated vesicles (CCVs) \\
\hline \multicolumn{3}{|c|}{ Cisplatin upregulated } \\
\hline 0.0009 & REACT_I3723 & Neurotransmitter release cycle \\
\hline 0.0011 & REACT_I665 & Glucagon signaling in metabolic regulation \\
\hline 0.0013 & REACT_12079 & PLC-gammal signalling \\
\hline 0.0057 & REACT_9053 & CaM pathway \\
\hline 0.0101 & REACT_I505 & Integration of energy metabolism \\
\hline 0.0141 & REACT_I5333 & Adenylate cyclase inhibitory pathway \\
\hline 0.0215 & REACT_18312 & NCAMI interactions \\
\hline 0.0238 & REACT_I5497 & PKA-mediated phosphorylation of CREB \\
\hline
\end{tabular}

species by intracellular-reducing agents such as ascorbic acid and glutathione. Furthermore, exposure to $0.1 \mathrm{M} \mathrm{HCl}$, representing gastric acidity, resulted in two-fold increased antiproliferative activity. ${ }^{16}$ Reduction/activation of oxoplatin at a low $\mathrm{pH}$ is an advantage for targeted release in the acidic microenvironment of solid tumors.

Although 40 years have passed since the discovery of the anticancer activity of cisplatin, the mechanism of action of platinum complexes is still unclear. ${ }^{24}$ The question of whether platinum(IV) compounds have intrinsic activity or whether they serve as prodrugs that are reduced to platinum(II) molecules before reaching their DNA target remains to be resolved. Platinum(IV)-ammine complexes containing the chelating ligand 1,2-diaminocyclohexane combined with a variety of coordinating anions were found to react with 9-methylxanthine, 9-methylhypoxanthine, and guanosine-5' monophosphate, providing evidence that not all platinum(IV) compounds represent prodrugs. ${ }^{25}$ Oxoplatin is capable of forming DNA adducts in a rather slow process. ${ }^{15}$ Oxoplatin was furthermore found to accumulate in tumor tissue, and metabolization resulted in the formation of several species, amongst them cisplatin, pointing to the role of oxoplatin as a prodrug of cisplatin; however, this hypothesis has not been validated so far. Oxoplatin reacted with $0.1 \mathrm{M} \mathrm{HCl}$ as well as DATCP(IV) yielded identical infrared spectra and cytotoxic effects. ${ }^{16}$ The reduction potential of the platinum-based drugs depends mainly on the axial ligands, with chloride substituents reduced most easily, hydroxide groups most stable, and carboxylate ligands lying between the two extremes. ${ }^{23,26}$

According to the ATCC, the NCI-H526 cell line, originating from a bone metastasis of an SCLC patient prior to therapy, expresses neuron-specific enolase, brain enzyme of creatine kinase, and p53 mRNA. Comparison of the gene expression patterns of control and treated NCI-H526 cells revealed significant differences in the expression pattern of target genes for cisplatin and DATCP(IV). Cisplatin-downregulated transcripts are involved in glutathione conjugation, pyruvate metabolism and citric acid cycle, cell signal transduction and metabolism of a range of carbohydrates and amino acids, and upregulation of pathways employed in metabolic regulation, energy metabolism, and cell signaling in NCI-H526 cells, which point to a restricted and selective cellular response. 
Table 3 Over-representation pathway analysis of genes more than four-fold down- or upregulated in $\mathrm{NCl}$ - $\mathrm{H} 526$ small cell lung cancer cells treated with DATCP(IV). Gene expression was assessed using Applied Biosystems Human Genome Survey Microarray V2.0, and data were analyzed using the Reactome database

\begin{tabular}{|c|c|c|}
\hline$P$ value & Identifier event & Name of this event \\
\hline \multicolumn{3}{|c|}{ DATCP(IV) downregulated } \\
\hline$<0.001$ & REACT_I477 & Eukaryotic translation elongation \\
\hline$<0.001$ & REACT_I0I4 & Translation \\
\hline$<0.001$ & REACT_I70I5 & Metabolism of proteins \\
\hline$<0.001$ & REACT_7I & Gene expression \\
\hline$<0.001$ & REACT_6305 & Respiratory electron transport, ATP synthesis \\
\hline$<0.001$ & REACT_22393 & Respiratory electron transport \\
\hline$<0.001$ & REACT_6828 & APC/C-mediated degradation of cell cycle proteins \\
\hline$<0.001$ & REACT_21279 & Regulation of mitotic cell cycle \\
\hline 0.0001 & REACT_24994 & Regulation of mRNA stability \\
\hline 0.0001 & REACT_6954 & APC/C:Cdc20 degradation of mitotic proteins \\
\hline 0.0002 & REACT_25325 & Destabilization of mRNA by AUFI (hnRNP D0) \\
\hline 0.0003 & REACT_9029 & Cyclin A:Cdk2-associated events at S phase \\
\hline 0.0008 & REACT_383 & DNA replication \\
\hline 0.0008 & REACT_829 & Regulation of DNA replication \\
\hline 0.0010 & REACT_20605 & Metabolism of mRNA \\
\hline 0.0011 & REACT_2014 & Synthesis of DNA \\
\hline 0.0013 & REACT_1625 & p53-dependent GI DNA damage response \\
\hline 0.0056 & REACT_2160 & p53-independent DNA damage response \\
\hline 0.0121 & REACT_I383 & Glycolysis \\
\hline $0.028 \mathrm{I}$ & REACT_19195 & Adherens junctions interactions \\
\hline 0.0306 & REACT_I520 & Gluconeogenesis \\
\hline 0.0321 & REACT_578 & Apoptosis \\
\hline 0.0426 & REACT_6759 & Formation of ATP by chemiosmotic coupling \\
\hline 0.0436 & REACT_474 & Metabolism of carbohydrates \\
\hline \multicolumn{3}{|c|}{ DATCP(IV) upregulated } \\
\hline 0.0001 & REACT_18425 & Prostanoid ligand receptors \\
\hline 0.0021 & REACT_I3705 & Phase I - functionalization of compounds \\
\hline 0.0036 & REACT_20582 & Zinc efflux and compartmentalization by the SLC30 family \\
\hline 0.0045 & REACT_20547 & Metal ion SLC transporters \\
\hline 0.0048 & REACT_7963 & Packaging of telomere ends \\
\hline 0.0048 & REACT_13433 & Biological oxidations \\
\hline 0.0091 & REACT_19305 & Transport of glucose, bile salts, metal ions, and amine compounds \\
\hline 0.0189 & REACT_19118 & SLC-mediated transmembrane transport \\
\hline 0.0243 & REACT_23928 & SOSI activates H-Ras \\
\hline 0.0324 & REACT_20676 & Cell junction organization \\
\hline 0.0385 & REACT_24024 & Gab2 binds the p85 subunit of class IA PI3 kinases \\
\hline
\end{tabular}

In contrast, DATCP(IV) suppresses expression of a host of genes participating in many aspects of cellular processes like protein synthesis and turnover, replication, transcription, respiration, cell cycle regulation, p53-dependent and -independent damage response, and glycolysis/gluconeogenesis. Upregulated transcription of genes involved in the metabolism of xenobiotics and metal ion transport seems to be important in drug resistance. The finding of only one single overlapping pathway, namely "metabolism of carbohydrates and glycolysis/gluconeogenesis", corroborates the fundamental differences in the mechanisms of cytotoxicity induced by the two platinum compounds and contradicts the exclusive role of DATCP(IV) as a prodrug of cisplatin.
All platinum(IV) complexes that have reached clinical trials thus far have yielded a platinum(II) central atom, reductively formed in vivo by endogenous molecules such as glutathione and ascorbate. ${ }^{5}$ Unexpectedly, the extracellular reduction of ormaplatin was primarily accomplished by protein sulfhydryl groups but not by glutathione, predominantly leading to the expected cis-dichlorido-(D,L-trans)-1, 2-diamineplatinum(II) among other products, due to substitution of chloride ligands. ${ }^{27}$ In clinical trials of ormaplatin, approximately $60 \%$ of the platinum in blood was bound to proteins ( $50 \%$ irreversibly) at the end of infusion, and the drug exhibited severe and unpredictable neurotoxicity. ${ }^{17,28-31}$ Similarly, the reaction of 
iproplatin with glutathione yielded cis-di(isopropylamine) chlorido-glutathionato-platinum(II) and not the expected cis-dichlorido-species. Therefore, binding of one of the available coordination sites of this platinum(II) product to glutathione precludes the formation of bifunctional adducts with DNA. ${ }^{29,32}$ Reaction with cysteine-rich cellular proteins and zinc-finger transcription factors as well as disruption of protein-DNA complexes may represent alternative targets for this iproplatin metabolite. ${ }^{33,34}$ The intracellular fate of DATCP(IV) has not been clarified so far, but analogically to the platinum complexes ormaplatin and iproplatin as well as in accordance with the present study, DATCP(IV) seems to bind to a host of cellular proteins, which results in shutdown of their transcription, rather than conversion to free platinum(II) compounds and DNA damage preceding impairment of transcription.

\section{Conclusion}

Here we demonstrate that the effects of DATCP(IV) on global gene expression of an SCLC cell line differ fundamentally from those of cisplatin. It is concluded that the metabolite itself, or intracellular reaction products thereof, impair a host of important proteins, resulting in the shutdown of a whole panel of genes involved in pathways effecting metabolism of cellular constituents and energy production. Thus, our data suggest that this compound may act as an anticancer drug originally and not by serving only as a prodrug of cisplatin, as was previously deduced from its chemical properties, such as the reduction potentials of tetrachlorido-platinum(IV) complexes. ${ }^{25}$

\section{Acknowledgment}

This study was supported by a fund from the Jubiläumsfonds (National Bank of Austria, Grant No. 13345). We thank Dr Zoser B Salama of IPSS, Berlin, Germany, for kindly providing the chemicals oxoplatin and DATCP(IV) as well as for helpful discussion.

\section{Disclosure}

The authors report no conflict of interest associated with this work.

\section{References}

1. Kostova I. Platinum complexes as anticancer agents. Recent Patents Anti-Cancer Drug Discov. 2006;1(1):1-22.

2. Kelland L. The resurgence of platinum-based cancer chemotherapy. Nature Rev Cancer. 2007;7(8):573-584.

3. Wang D, Lippard SJ. Cellular processing of platinum anticancer drugs. Nature Rev Drug Discov. 2005;4(4):307-320.

4. Olszewski U, Hamilton G. A better platinum-based anticancer drug yet to come? Anticancer Agents Med Chem. 2010;10(4):293-301.
5. Hall MD, Mellor HR, Callaghan R, Hambley TW. Basis for design and development of platinum(IV) anticancer complexes. J Med Chemistry. 2007;50(15):3403-3411.

6. Hall MD, Alderden RA, Zhang M, et al. The fate of platinum(II) and platinum(IV) anti-cancer agents in cancer cells and tumours. $J$ Struct Biol. 2006;155:38-44.

7. Bhargava A, Vaishampayan UN. Satraplatin: leading the new generation of oral platinum agents. Expert Opin Investig Drugs. 2009;18(11):1787-1797.

8. Hambley TW, Battle AR, Deacon GB, et al. Modifying the properties of platinum(IV) complexes in order to increase biological effectiveness. J Inorg Biochem. 1999;77(1-2):3-12.

9. Foltinová V, Švihálková Šindlerová L, Horváth V, et al. Mechanisms of effects of platinum (II) and (IV) complexes. Comparison of cisplatin and oxaliplatin with satraplatin and LA-12, new PT(IV)-based drugs. Scripta Medica Facultatis Medicae Universitatis Brunensis Masarykianae. 2008;81:105-116.

10. Nakai T, Ando M, Okamoto Y, et al. Modulation of oxidative DNA damage and DNA-crosslink formation induced by cis-diamminetetrachloro-platinum(IV) in the presence of endogenous reductants. J Inorg Biochem. 2011;105(1):1-5.

11. Cubo L, Hambley TW, Sanz Miguel PJ, et al. The preparation and characterization of trans-platinum(IV) complexes with unusually high cytotoxicity. Dalton Trans. 2011;40:344-347.

12. Carr JL, Tingle MD, McKeage MJ. Satraplatin activation by haemoglobin, cytochrome $\mathrm{C}$ and liver microsomes in vitro. Cancer Chemother Pharmacol. 2006;57:483-490.

13. Konovalova AL, Presnov MA, Zheligovskaia NN, Treshchalina EM Antitumor effect of complex compounds of tetravalent platinum. Doklady Akademii Nauk SSSR. 1977;234(1):223-226.

14. Presnov MA, Konovalova AL, Kozlov AM, et al. The antitumor activity of oxoplatinum. Neoplasma. 1985;32(1):73-83.

15. Nováková $\mathrm{O}$, Vrána $\mathrm{O}$, Kiseleva VI, Brabec V. DNA interactions of antitumor platinum(IV) complexes. Eur J Biochem. 1995;228: 616-624.

16. Olszewski U, Ach F, Ulsperger E, et al. In vitro evaluation of oxoplatin: an oral platinum(IV) anticancer agent. Met Based Drugs. 2009:348916.

17. Schilder RJ, LaCreta FP, Perez RP, et al. Phase I and pharmacokinetic study of ormaplatin (tetraplatin, NSC 363812) administered on a day 1 and day 8 schedule. Cancer Res. 1994;54(3):709-717.

18. Trask C, Silverstone A, Ash CM, et al. A randomized trial of carboplatin versus iproplatin in untreated advanced ovarian cancer. J Clin Oncol. 1991;9(7):1131-1137.

19. Choi S, Filotto C, Bisanzo M, et al. Reduction and anticancer activity of platinum(IV) complexes. Inorg Chem. 1998;37:2500-2504.

20. Platts JA, Ermondi G, Caron G, et al. Molecular and statistical modeling of reduction peak potential and lipophilicity of platinum(IV) complexes. J Biol Inorg Chem. 2011;16(3):361-372.

21. Montaña AM, Batalla C. The rational design of anticancer platinum complexes: the importance of the structure-activity relationship. Curr Med Chem. 2009;16(18):2235-2260.

22. Galanski M. Recent developments in the field of anticancer platinum complexes. Recent Pat Anticancer Drug Discov. 2006;1(2):285-295.

23. Harper BW, Krause-Heuer AM, Grant MP, et al. Advances in platinum chemotherapeutics. Chemistry. 2010;16:7064-7077.

24. Gibson D. The mechanism of action of platinum anticancer agents: what do we really know about it? Dalton Trans. 2009;48:10681-10689.

25. Talman EG, Kidani Y, Mohrmann L, Reedijk J. Can Pt(IV)-amine complexes act as 'prodrugs'? Inorg Chim Acta. 1998;283:251-255.

26. Reithofer MR, Bytzek AK, Valiahdi SM, et al. Tuning of lipophilicity and cytotoxic potency by structural variation of anticancer platinum(IV) complexes. J Inorg Biochem. 2011;105:46-51.

27. Chaney SG, Gibbons GR, Wyrick SD, Podhasky P. An unexpected biotransformation pathway for tetrachloro-(d,1-trans)-1,2diaminocyclohexaneplatinum(IV) (tetraplatin) in the L1210 cell line. Cancer Res. 1991;51:969-973.

28. Pendyala L, Walsh JR, Huq MM, et al. Uptake and metabolism of iproplatin in murine L1210 cells. Cancer Chemother Pharmacol. 1989;25:15-18. 
29. Pendyala L, Arakali AV, Sansone P, et al. DNA binding of iproplatin and its divalent metabolite cis-dichloro-bis-isopropylamine platinum (II). Cancer Chemother Pharmacol. 1990;27:248-250.

30. McKeage MJ, Boxall FE, Jones M, Harrap KR. Lack of neurotoxicity of oral bisacetatoamminedichlorocyclohexylamine-platinum(IV) in comparison to cisplatin and tetraplatin in the rat. Cancer Res. 1994;54:629-631.

31. O'Rourke TJ, Weiss GR, New P, et al. Phase I clinical trial of ormaplatin (tetraplatin, NSC 363812). Anticancer Drugs. 1994;5(5):520-526.
32. Volckova E, Weaver E, Bose RN. Insight into the reactive form of the anticancer agent iproplatin. Eur J Med Chem. 2008;43:1081-1084.

33. Eastman A. Glutathione-mediated activation of anticancer platinum(IV) complexes. Biochem Pharmacol. 1987;36(23):4177-4178.

34. Kido Y, Khokhar AR, Siddik ZH. Glutathione-mediated modulation of tetraplatin activity against sensitive and resistant tumor cells. Biochemical Pharmacol. 1994;47:1635-1642.

\section{Publish your work in this journal}

The Journal of Experimental Pharmacology is an international, peerreviewed, open access journal publishing original research, reports, reviews and commentaries on all areas of laboratory and experimental pharmacology. The manuscript management system is completely online and includes a very quick and fair peer-review system.
Visit http://www.dovepress.com/testimonials.php to read real quotes from published authors. 